\title{
Metal-Specific Response of High-Resolution ICP-MS for Proteins Binding to Gold Nanoparticles in Human Serum
}

\author{
Olga V. Kuznetsova ${ }^{a}$, Nikolai G. Khlebtsov, ${ }^{\text {b,c }}$ Maciej Jarosz ${ }^{d}$, and Andrei R. Timerbaeva* \\ aVernadsky Institute of Geochemistry and Analytical Chemistry, 119991 Moscow, Russian \\ Federation \\ ${ }^{b}$ Institute of Biochemistry and Physiology of Plants and Microorganisms, 410049 Saratov, \\ Russian Federation \\ 'Saratov State University, 410012 Saratov, Russian Federation \\ ${ }^{d}$ Chair of Analytical Chemistry, Warsaw University of Technology, 00664 Warsaw, Poland \\ "Email: andrei.timerbaev@univie.ac.at
}

\section{TABLE OF CONTENTS}

Experimental part

Figure S1, TEM images of AuNPs

Figure S2, size distribution of AuNPs measured by DLS

Table S1, ICP-SFMS measurement parameters

Table S2, elemental composition of human serum

Table S3, effect of the centrifugation time on AuNP separation

Table S4-S7, lists of the most abundant proteins identified by LC-MS/MS 


\section{EXPERIMENTAL PART}

Reagents and Materials. The AuNPs were prepared and characterized as published before (see Figures S1 and S2, for TEM and DLS data). Each type of the particles was suspended in PBS to give $0.1 \mathrm{mg} \mathrm{L}^{-1} \mathrm{Au}$ in final suspension. All reagents were of analytical grade and products of Sigma-Aldrich (St. Louis, MO, USA). Pooled human serum (derived from plasma collected from healthy $\mathrm{AB}$ blood group male donors) was provided by National Medicinal Research Center of Hematology, Moscow, Russian Federation, and stored at -80 ${ }^{\circ} \mathrm{C}$ before analysis. High purity water obtained from a NANO Pure purifier system (Thermo Fisher Scientific, Waltham, MA, USA) was used. For ultrafiltration experiments, 30 and 100 kDa cut-off filters (Amicon Ultracel; Millipore, Molsheim, France) were employed.

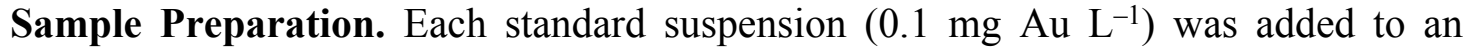
equal volume of the standard solution of human serum and the mixture (corresponding to 10fold diluted serum and containing $50 \mu \mathrm{g} \mathrm{L}-1 \mathrm{Au}$ ) was incubated at $37^{\circ} \mathrm{C}$. An aliquot was taken after 2, 5, 10, 20, and $60 \mathrm{~min}$, placed into a centrifugal tube, and centrifuged for $10 \mathrm{~min}$ at $10,000 \mathrm{rcf}$ at $0{ }^{\circ} \mathrm{C}$. Supernatant was isolated and its aliquot was placed into an ultracentrifugal unit $(30 \mathrm{kDa})$ and centrifuged for $15 \mathrm{~min}$ at 10,000 rcf. Supernatant and filtrate were diluted (1:10) with $3 \% \mathrm{HNO}_{3}$ and subjected to analysis. For each sort of AuNPs a blank sample was run by repeating the same procedure without serum (i.e. PBS instead of serum solution) to assess the background levels of each element. All samples were triplicated to assess the experimental reproducibility.

In additional experimental series, the protein-coated AuNPs, remaining on a $100 \mathrm{kDa}$ membrane filter after ultrafiltration, were isolated by reverse ultrafiltration. Then they were directly analyzed (after dilution (1:10) with $3 \% \mathrm{HNO}_{3}$ ) or subjected to repeat rinsing with PBS (each time for $10 \mathrm{~min}$ ). Rinsates (freed from nanoparticles) and the remaining particles were similarly diluted and analyzed.

For the identification of AuNP-binding proteins, the protein conjugates, isolated by centrifugation and washed (two times) with PBS, were treated with $2 \%$ SDS in PBS upon ultrasound agitation for $30 \mathrm{~min}$. After centrifugation, the supernatant was collected and the same treatment repeated. The combined supernatant was dried at $95{ }^{\circ} \mathrm{C}$ and the residue was submitted to standard proteomic analysis (see below).

ICP-SFMS Analysis. An Element 2 instrument (Thermo Fisher Scientific) was used for ICP-SFMS measurements. The total metal concentrations in acidified samples were determined using the instrumental settings collected in Table S1. External calibration was 
done against calibration curves prepared using one- or multi-element standard solutions (High-Purity Standards, North Charleston, SC, USA) in the range of $0.1-100 \mu \mathrm{g} \mathrm{L}^{-1}$, and In was used as an internal standard to monitor instrumental plasma stability.

LC-MS/MS Analysis. After customary steps of reduction, acetylation and trypsinization of proteins and sample purification on $\mathrm{C} 18$ spin columns, the resulting peptide mixtures were separated by $\mathrm{C} 18$ reverse phase LC using an EASY-nLC ${ }^{\text {TM }} 1000$ UHPLC system (Thermo Fisher Scientific) on-line connected to a Q Exactive HF mass spectrometer (Thermo Fisher Scientific). Data files were processed using a PEAKS X proteomic machine. The identified proteins are listed in Tables S4-S7.

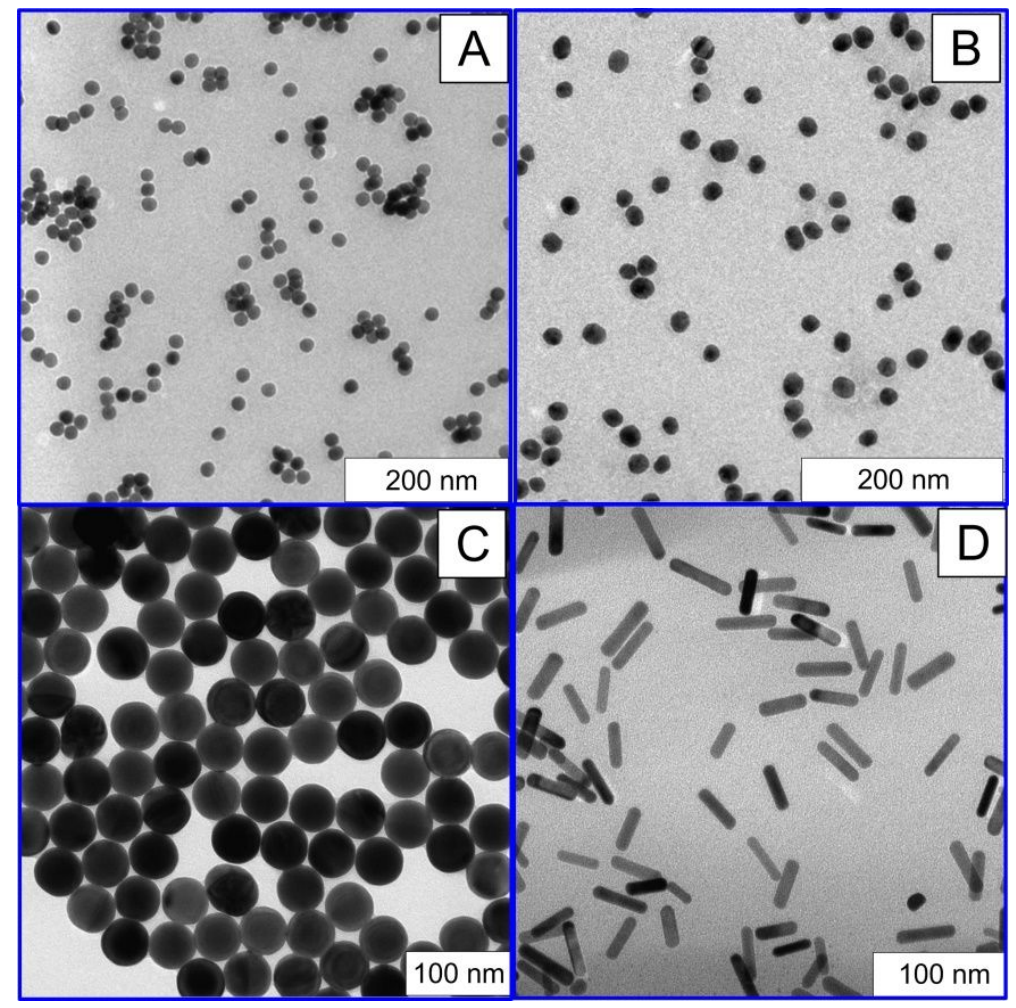

Figure S1. TEM images of (A) AuNP-1; (B) AuNP-2; (C) AuNP-3 and (D) AuNP-4. 


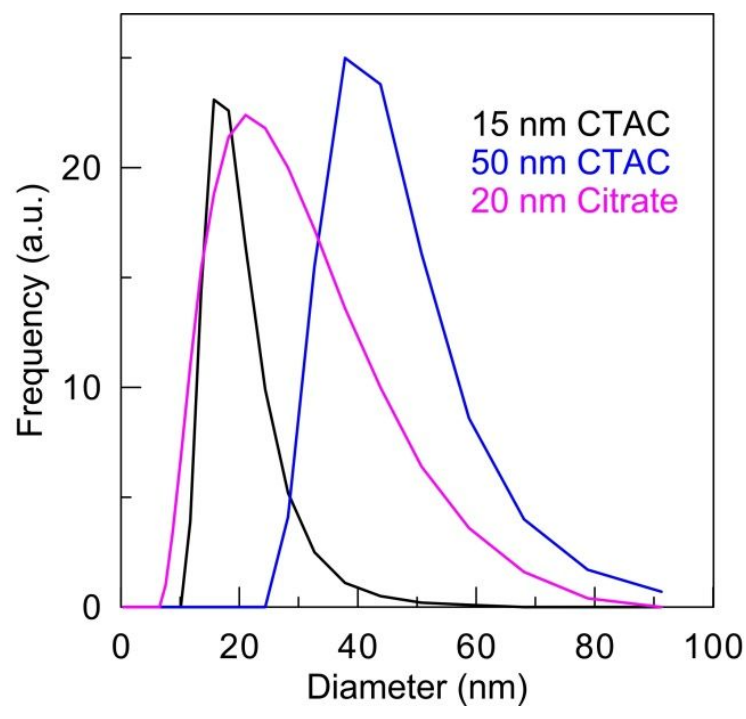

Figure S2. Size distribution of AuNPs measured by DLS. 
Table S1. ICP-SFMS Measurement Parameters

\begin{tabular}{|c|c|}
\hline parameter & setting \\
\hline plasma gas flow & $16 \mathrm{~L} \mathrm{~min}^{-1}$ \\
\hline auxiliary gas flow & $0.73 \mathrm{~L} \mathrm{~min}^{-1}$ \\
\hline analyzed sample flow & $0.78 \mathrm{~mL} \mathrm{~min}^{-1}$ \\
\hline RF power & $1250 \mathrm{~W}$ \\
\hline dwell time & $10 \mathrm{~ms}$ \\
\hline $\begin{array}{c}\text { isotopes measured } \\
\left(\mathrm{LOD}, \mu \mathrm{g} \mathrm{L}^{-1}\right)\end{array}$ & $\begin{array}{l}{ }^{59} \mathrm{Co}(0.006),{ }^{52} \mathrm{Cr}(0.028),{ }^{63} \mathrm{Cu} \\
(0.069),{ }^{56} \mathrm{Fe}(0.19),{ }^{60} \mathrm{Ni} \\
(0.10),{ }^{55} \mathrm{Mn}(0.06),{ }^{51} \mathrm{~V} \\
(0.044),{ }^{66} \mathrm{Zn}(2.0) \text { (medium } \\
\text { resolution mode) } \\
{ }^{197} \mathrm{Au}(0.03) \text { (low resolution } \\
\text { mode) }\end{array}$ \\
\hline internal standard & ${ }^{115} \mathrm{In}$ \\
\hline
\end{tabular}

Table S2. Metal Concentrations $\left(\mu \mathrm{g} \mathrm{L}^{-1} ; n=3\right)$ in Human Serum under Investigation

\begin{tabular}{|c|c|c|}
\hline metal & total & $\mathrm{LMW}^{a}$ \\
\hline $\mathrm{Fe}$ & $416.7 \pm 0.3$ & $208.5 \pm 5.7$ \\
\hline $\mathrm{Cu}$ & $314.0 \pm 5.6$ & $146.1 \pm 1.0$ \\
\hline $\mathrm{Zn}$ & $230.7 \pm 0.5$ & $228.5 \pm 2.0$ \\
\hline $\mathrm{Ni}$ & $3.0 \pm 0.2$ & $3.1 \pm 0.1$ \\
\hline $\mathrm{Mn}$ & $3.9 \pm 0.1$ & $3.9 \pm 0.2$ \\
\hline $\mathrm{Cr}$ & $2.0 \pm 0.2$ & $1.4 \pm 0.1$ \\
\hline $\mathrm{V}$ & $<$ LOD & $<\mathrm{LOD}$ \\
\hline $\mathrm{Co}$ & $<\mathrm{LOD}$ & $<\mathrm{LOD}$ \\
\hline
\end{tabular}

${ }^{a}$ In filtrate (see $C_{\mathrm{M}, \text { non-protein }}$ in Eq. 1). 
Table S3. Effect of the Centrifugation Time on AuNP Separation

\begin{tabular}{|c|c|c|}
\hline \multirow{2}{*}{$\begin{array}{c}\text { time } \\
(\mathrm{min})\end{array}$} & \multicolumn{2}{|c|}{$c_{\mathrm{Au}}\left(\mu \mathrm{g} \mathrm{L}^{-1} ; n=3\right)$} \\
\cline { 2 - 3 } & AuNP-1 & AuNP-4 \\
\hline $0^{a}$ & $98.5 \pm 1.2$ & $97.1 \pm 1.3$ \\
\hline 2 & $50.5 \pm 0.8$ & $73.0 \pm 1.0$ \\
\hline 5 & $38.2 \pm 0.5$ & $38.1 \pm 0.8$ \\
\hline 10 & $6.7 \pm 0.4$ & $7.1 \pm 0.5$ \\
\hline 15 & $6.2 \pm 0.3$ & $7.2 \pm 0.3$ \\
\hline
\end{tabular}

${ }^{a}$ Standard suspensions $\left(0.1 \mathrm{mg} \mathrm{L}^{-1} \mathrm{Au}\right)$ without centrifugation.

Table S4. Most Abundant Proteins in the Corona of AuNP-1

\begin{tabular}{|c|l|c|}
\hline & \multicolumn{1}{|c|}{ protein } & coverage (\%) \\
\hline 1 & Albumin & 89 \\
\hline 2 & Apolipoprotein A & 88 \\
\hline 3 & Tubulin beta-1 chain & 84 \\
\hline 4 & Isoform smooth muscle of myosin & 83 \\
\hline 5 & Hemoglobin subunit beta & 80 \\
\hline 6 & Immunoglobulin kappa constant & 79 \\
\hline 7 & Immunoglobulin lambda constant 3 & 79 \\
\hline 8 & Immunoglobulin lambda constant 2 & 72 \\
\hline 9 & Vitamin D-binding protein & 72 \\
\hline 10 & Isoform 3 of vitamin D-binding protein & 71 \\
\hline 11 & Fibrinogen beta chain & 71 \\
\hline 12 & Talin-1 & 71 \\
\hline 13 & Complement C3 & 71 \\
\hline 14 & Tubulin beta chain & 69 \\
\hline 15 & Tubulin beta-4B chain & \\
\hline 16 & Alpha-actinin-1 & 69 \\
\hline 17 & Apolipoprotein A-II & 86 \\
\hline 18 & Isoform 2 of alpha-actinin-1 & 89 \\
\hline
\end{tabular}




\begin{tabular}{|c|c|c|}
\hline 19 & Beta-2-glycoprotein 1 & 68 \\
\hline 20 & Apolipoprotein E & 68 \\
\hline 21 & Isoform gamma-A of fibrinogen gamma chain & 67 \\
\hline 22 & Immunoglobulin kappa joining 1 & 67 \\
\hline 23 & Isoform 3 of alpha-actinin-1 & 66 \\
\hline 24 & Serotransferrin & 66 \\
\hline 25 & Alpha-1-antitrypsin & 66 \\
\hline 26 & Actin cytoplasmic 2 & 66 \\
\hline 27 & Actin cytoplasmic 1 & 66 \\
\hline 28 & Fibrinogen gamma chain & 65 \\
\hline 29 & Transthyretin & 65 \\
\hline 30 & Isoform 2 of filamin-A & 64 \\
\hline 31 & Filamin-A & 64 \\
\hline 32 & Apolipoprotein A-IV & 64 \\
\hline 33 & Hemoglobin subunit alpha & 64 \\
\hline 34 & Myosin-9 & 63 \\
\hline 35 & Myosin regulatory light chain $12 \mathrm{~A}$ & 63 \\
\hline 36 & Hemoglobin subunit delta & 63 \\
\hline 37 & Apolipoprotein B-100 & 62 \\
\hline 38 & Keratin type I cytoskeletal 9 & 62 \\
\hline 39 & Myosin regulatory light chain $12 \mathrm{~B}$ & 62 \\
\hline 40 & Isoform 4 of superoxide dismutase & 62 \\
\hline 41 & Hemopexin & 61 \\
\hline 42 & Tropomyosin alpha-4 chain & 61 \\
\hline 43 & Ceruloplasmin & 60 \\
\hline 44 & Immunoglobulin heavy constant mu & 60 \\
\hline 45 & Isoform 2 of tubulin alpha-4A chain & 60 \\
\hline 46 & Integrin beta- 3 & 59 \\
\hline 47 & Plasminogen & 59 \\
\hline 48 & Keratin type I cytoskeletal 10 & 59 \\
\hline 49 & Apolipoprotein C-II & 59 \\
\hline 50 & Thrombospondin-1 & 58 \\
\hline
\end{tabular}


Table S5. Most Abundant Proteins in the Corona of AuNP-2

\begin{tabular}{|c|c|c|}
\hline & protein & coverage $(\%)$ \\
\hline 1 & Albumin & 90 \\
\hline 2 & Isoform smooth muscle of myosin & 84 \\
\hline 3 & Apolipoprotein A & 82 \\
\hline 4 & Immunoglobulin kappa constant & 80 \\
\hline 5 & Tubulin beta-1 chain & 79 \\
\hline 6 & Serotransferrin & 78 \\
\hline 7 & Hemoglobin subunit beta & 78 \\
\hline 8 & Immunoglobulin lambda constant 3 & 77 \\
\hline 9 & Isoform gamma-A of fibrinogen gamma chain & 73 \\
\hline 10 & Fibrinogen beta chain & 72 \\
\hline 11 & Hemoglobin subunit alpha & 72 \\
\hline 12 & Complement C3 & 71 \\
\hline 13 & Fibrinogen gamma chain & 71 \\
\hline 14 & Tubulin beta chain & 71 \\
\hline 15 & Tubulin beta-4B chain & 71 \\
\hline 16 & Actin cytoplasmic 1 & 70 \\
\hline 17 & Actin cytoplasmic 2 & 70 \\
\hline 18 & Vitamin D-binding protein & 69 \\
\hline 19 & Transthyretin & 69 \\
\hline 20 & Apolipoprotein A-II & 69 \\
\hline 21 & Ceruloplasmin & 67 \\
\hline 22 & Alpha-1-antitrypsin & 67 \\
\hline 23 & Isoform 3 of vitamin D-binding protein & 67 \\
\hline 24 & Hemopexin & 67 \\
\hline 25 & Plasminogen & 67 \\
\hline 26 & Retinol-binding protein 4 & 67 \\
\hline 27 & Immunoglobulin kappa joining 1 & 67 \\
\hline 28 & Apolipoprotein A-IV & 66 \\
\hline 29 & Immunoglobulin heavy constant gamma 4 & 65 \\
\hline 30 & Hemoglobin subunit delta & 65 \\
\hline
\end{tabular}




\begin{tabular}{|c|c|c|}
\hline 31 & Haptoglobin & 63 \\
\hline 32 & Keratin type II cytoskeletal 2 epidermal & 63 \\
\hline 33 & Apolipoprotein B-100 & 62 \\
\hline 34 & Isoform 2 of tubulin alpha-1A chain & 62 \\
\hline 35 & Haptoglobin-related protein & 62 \\
\hline 36 & Myosin regulatory light polypeptide 9 & 62 \\
\hline 37 & Talin-1 & 61 \\
\hline 38 & Isoform 2 of filamin-A & 61 \\
\hline 39 & Filamin-A & 61 \\
\hline 40 & Isoform 2 of tubulin alpha- $4 \mathrm{~A}$ chain & 61 \\
\hline 41 & Serum paraoxonase/arylesterase 1 & 61 \\
\hline 42 & Immunoglobulin heavy constant gamma 2 & 60 \\
\hline 43 & Tubulin alpha-4A chain & 59 \\
\hline 44 & Immunoglobulin kappa light chain & 59 \\
\hline 45 & Apolipoprotein C-II & 59 \\
\hline 46 & Complement factor $\mathrm{H}$ & 58 \\
\hline 47 & Isoform 2 of fibrinogen alpha chain & 58 \\
\hline 48 & Immunoglobulin heavy constant mu & 58 \\
\hline 49 & Tubulin alpha-1C chain & 58 \\
\hline 50 & Keratin type I cytoskeletal 14 & 58 \\
\hline
\end{tabular}


Table S6. Most Abundant Proteins in the Corona of AuNP-3

\begin{tabular}{|c|c|c|}
\hline & protein & coverage $(\%)$ \\
\hline 1 & Albumin & 87 \\
\hline 2 & Isoform gamma-A of fibrinogen gamma chain & 85 \\
\hline 3 & Complement C3 & 83 \\
\hline 4 & Hemoglobin subunit beta & 83 \\
\hline 5 & Fibrinogen gamma chain & 82 \\
\hline 6 & Apolipoprotein A-I & 80 \\
\hline 7 & Immunoglobulin kappa constant & 80 \\
\hline 8 & Fibrinogen beta chain & 79 \\
\hline 9 & Retinol-binding protein 4 & 76 \\
\hline 10 & Serotransferrin & 75 \\
\hline 11 & Immunoglobulin lambda constant 3 & 75 \\
\hline 12 & Immunoglobulin lambda constant 2 & 75 \\
\hline 13 & C4b-binding protein alpha chain & 73 \\
\hline 14 & Apolipoprotein B-100 & 72 \\
\hline 15 & Actin cytoplasmic 1 & 70 \\
\hline 16 & Actin cytoplasmic 2 & 70 \\
\hline 17 & Ceruloplasmin & 69 \\
\hline 18 & Alpha-2-macroglobulin & 69 \\
\hline 19 & Fibrinogen alpha chain & 69 \\
\hline 20 & Transthyretin & 69 \\
\hline 21 & Apolipoprotein A-II & 69 \\
\hline 22 & Vitamin D-binding protein & 68 \\
\hline 23 & Serum paraoxonase/arylesterase 1 & 68 \\
\hline 24 & Complement C4-B & 67 \\
\hline 25 & Complement C4-A & 67 \\
\hline 26 & Alpha-1-antitrypsin & 67 \\
\hline 27 & Immunoglobulin kappa joining 1 & 67 \\
\hline 28 & Isoform 3 of vitamin D-binding protein & 65 \\
\hline 29 & Apolipoprotein A-IV & 65 \\
\hline 30 & Haptoglobin-related protein & 65 \\
\hline
\end{tabular}




\begin{tabular}{|c|c|c|}
\hline 31 & Hemoglobin subunit alpha & 64 \\
\hline 32 & Hemoglobin subunit delta & 64 \\
\hline 33 & Immunoglobulin heavy constant mu & 63 \\
\hline 34 & Apolipoprotein E & 62 \\
\hline 35 & Haptoglobin & 61 \\
\hline 36 & CD5 antigen-like & 61 \\
\hline 37 & Tubulin beta-1 chain & 61 \\
\hline 38 & Immunoglobulin heavy constant alpha 1 & 61 \\
\hline 39 & Isoform 1 of fibronectin & 59 \\
\hline 40 & Keratin type I cytoskeletal 9 & 59 \\
\hline 41 & Immunoglobulin heavy constant gamma 2 & 59 \\
\hline 42 & Isoform 2 of haptoglobin-related protein & 59 \\
\hline 43 & Apolipoprotein C-II & 59 \\
\hline 44 & Prothrombin & 57 \\
\hline 45 & Hemopexin & 57 \\
\hline 46 & Apolipoprotein C-III & 56 \\
\hline 47 & Complement C1r subcomponent & 55 \\
\hline 48 & Keratin type I cytoskeletal 10 & 55 \\
\hline 49 & Isoform LMW of kininogen-1 & 55 \\
\hline 50 & Isoform 2 of tubulin alpha- $4 \mathrm{~A}$ chain & 55 \\
\hline
\end{tabular}


Table S7. Most Abundant Proteins in the Corona of AuNP-4

\begin{tabular}{|c|c|c|}
\hline & protein & coverage $(\%)$ \\
\hline 1 & Albumin & 91 \\
\hline 2 & Isoform smooth muscle of myosin light & 84 \\
\hline 3 & Hemoglobin subunit beta & 83 \\
\hline 4 & Immunoglobulin kappa constant & 80 \\
\hline 5 & Tubulin beta-1 chain & 78 \\
\hline 6 & Apolipoprotein A-I & 77 \\
\hline 7 & Vitamin D-binding protein & 76 \\
\hline 8 & Immunoglobulin lambda constant 3 & 75 \\
\hline 9 & Immunoglobulin lambda constant 2 & 75 \\
\hline 10 & Complement C3 & 74 \\
\hline 11 & Fibrinogen beta chain & 74 \\
\hline 12 & Serotransferrin & 73 \\
\hline 13 & Isoform 3 of vitamin D-binding protein & 73 \\
\hline 14 & Isoform gamma-A of fibrinogen gamma chain & 73 \\
\hline 15 & Hemoglobin subunit alpha & 72 \\
\hline 16 & Fibrinogen gamma chain & 71 \\
\hline 17 & Actin cytoplasmic 1 & 71 \\
\hline 18 & Actin cytoplasmic 2 & 71 \\
\hline 19 & Tubulin beta chain & 71 \\
\hline 20 & Ceruloplasmin & 69 \\
\hline 21 & Hemopexin & 69 \\
\hline 22 & Transthyretin & 69 \\
\hline 23 & Apolipoprotein A-II & 69 \\
\hline 24 & Alpha-1-antitrypsin & 67 \\
\hline 25 & Haptoglobin & 67 \\
\hline 26 & Tubulin beta-4B chain & 67 \\
\hline 27 & Immunoglobulin kappa joining 1 & 67 \\
\hline 28 & Talin-1 & 63 \\
\hline 29 & Myosin regulatory light chain $12 \mathrm{~A}$ & 63 \\
\hline
\end{tabular}




\begin{tabular}{|l|l|c|}
\hline 30 & Isoform 2 of filamin-A & 62 \\
\hline 31 & Plasminogen & 62 \\
\hline 32 & Coagulation factor XI & 62 \\
\hline 33 & $\begin{array}{l}\text { LIM and senescent cell antigen-like-containing } \\
\text { domain protein 1 }\end{array}$ & 62 \\
\hline 34 & Apolipoprotein E & 62 \\
\hline 35 & Stomatin & 62 \\
\hline 36 & Myosin regulatory light chain 12B & 62 \\
\hline 37 & Myosin regulatory light polypeptide 9 & 62 \\
\hline 38 & Apolipoprotein B-100 & 61 \\
\hline 39 & Filamin-A & 61 \\
\hline 40 & Alpha-actinin-1 & 61 \\
\hline 41 & Immunoglobulin heavy constant gamma 2 & 60 \\
\hline 42 & Keratin type I cytoskeletal 10 & 60 \\
\hline 43 & Isoform 2 of tubulin alpha-4A chain & 59 \\
\hline 44 & Apolipoprotein C-II & 59 \\
\hline 45 & Isoform 4 of superoxide dismutase & 58 \\
\hline 46 & Myosin-9 & 58 \\
\hline 47 & Complement factor H & 58 \\
\hline 48 & Keratin type I cytoskeletal 9 & 58 \\
\hline 49 & Tubulin alpha-1C chain & 68 \\
\hline 50 & Tubulin alpha-4A chain & 6 \\
\hline & & 60 \\
\hline
\end{tabular}

